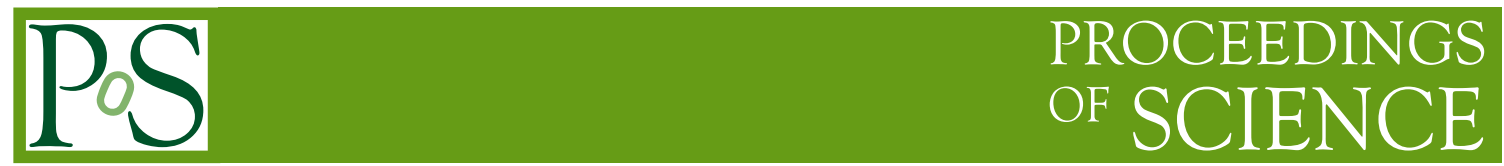

\title{
Long-Lived Particles at HL-LHC with the ATLAS detector
}

\author{
Cristiano Sebastiani, on behalf of the ATLAS collaboration \\ INFN Roma and Sapienza Università di Roma \\ E-mail: cristiano.sebastianiecern.ch
}

Several new physics models that extend the Standard Model require the existence of Long-Lived Particle (LLP) as a solution for the problems like Dark Matter and Naturalness. The ATLAS Phase-II upgrade detector and the expected large data set from high luminosity LHC upgrade offers an opportunity to probe the yet unexplored region of the phase space. For muon spectrometer based searches, neutral LLP decaying to collimated jets of leptons and light hadrons (lepton-jets) are of great interest. These particles offer an unique signature that can lead to an early discovery. New triggering techniques and algorithm have been developed and studied to improve the selection of highly boosted lepton-jets.

Sixth Annual Conference on Large Hadron Collider Physics (LHCP2018)

4-9 June 2018

Bologna, Italy 


\section{Introduction}

Most of the hidden sector models coupled to the visible sector predict the existence of longlived particles (LLP). The search for these particles depends on the LLP decay length, which can range from few $\mu \mathrm{m}$ to several meters outside the detector. In this study, only neutral long-lived particles which decay far from the interaction point are considered and therefore rely on the outermost ATLAS detector, the muon spectrometer (MS). These signatures represent a great challenge for both the trigger and the event reconstruction capabilities of the ATLAS detector [1], which is optimized for prompt searches. As a benchmark, the vector portal FRVZ [2] model is considered. The FRVZ model predicts the existence of a dark sector that is weakly coupled to the SM sector. As vector mediator, a dark photon is assumed. This model presents two light $400 \mathrm{MeV}$ dark photons, decaying to SM via kinetic mixing, produced by a $125 \mathrm{GeV}$ SM Higgs particle. At the LHC, these dark photons would typically be produced with large boost, due to their small mass, resulting in collimated structures containing pairs of leptons.

\section{Run2 level-1 di-muon trigger limitations}

The typical signature is a highly boosted object that can decay to collimated structures containing pairs of muons. There are two critical features in triggering these events:

- Low muon $p_{\mathrm{T}}$ threshold

- Small di-muon angular separation

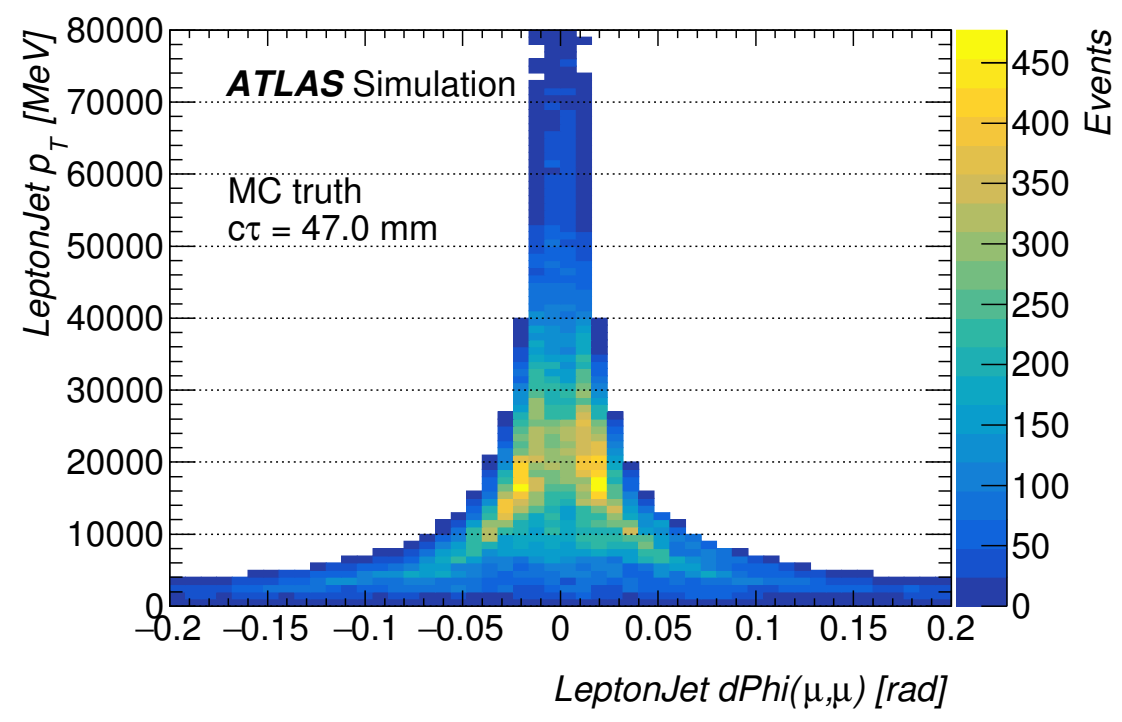

Figure 1: Truth transverse momentum distribution of the $\gamma_{\mathrm{d}}$, vector mediator, as a function of the opening angle in the $\Phi$ plane of the two outcoming muons, decay products of the $\gamma_{\mathrm{d}}$ [3]. A Monte Carlo sample with average LLP lifetime $\mathrm{c} \tau=49.23 \mathrm{~mm}$ is considered, resulting from Dark Sector models [2].

The selection of close-by muon candidates, requires a separation of $\Delta \eta \times \Delta \phi=0.2 \times 0.2$ between the two muon candidates. This results in large inefficiencies. The distribution of the 
vector portal mediator $p_{\mathrm{T}}$ vs. the opening angle between the two outgoing muons is shown in Fig. 1. These inefficiencies are also seen in many different searches like the B physics channel $\mathrm{B}_{\mathrm{S}} \rightarrow \mathrm{J} / \Psi \phi$, as can be seen in Fig. 2 .

As a possible improvement of the selections of these events, multiple trigger candidates in the same region of interest are considered. This would allow to choose a lower $p_{\mathrm{T}}$ threshold without increasing significantly the trigger rate.

\section{Phase-II level-0 new di-muon trigger}

In these highly boosted events, the out-coming muons from the dark photon processes are close-by and likely to fall in the same region of interest (RoI). The current system is able to select only one muon candidate per $\operatorname{RoI}(\Delta \eta \times \Delta \phi=0.2 \times 0.2)$, any information on muon multiplicity is lost. As can be seen in Fig. 1, most of the signal is in a range between 10 and $20 \mathrm{GeV}$ of the dark photon $p_{\mathrm{T}}$ and the muons fall in the same RoI. In the current setup, due to the high single muon trigger rate, the lowest possible muon trigger threshold is a $p_{\mathrm{T}}^{\text {thr }}=20 \mathrm{GeV}$ selection, and it will lose most of the signal events.
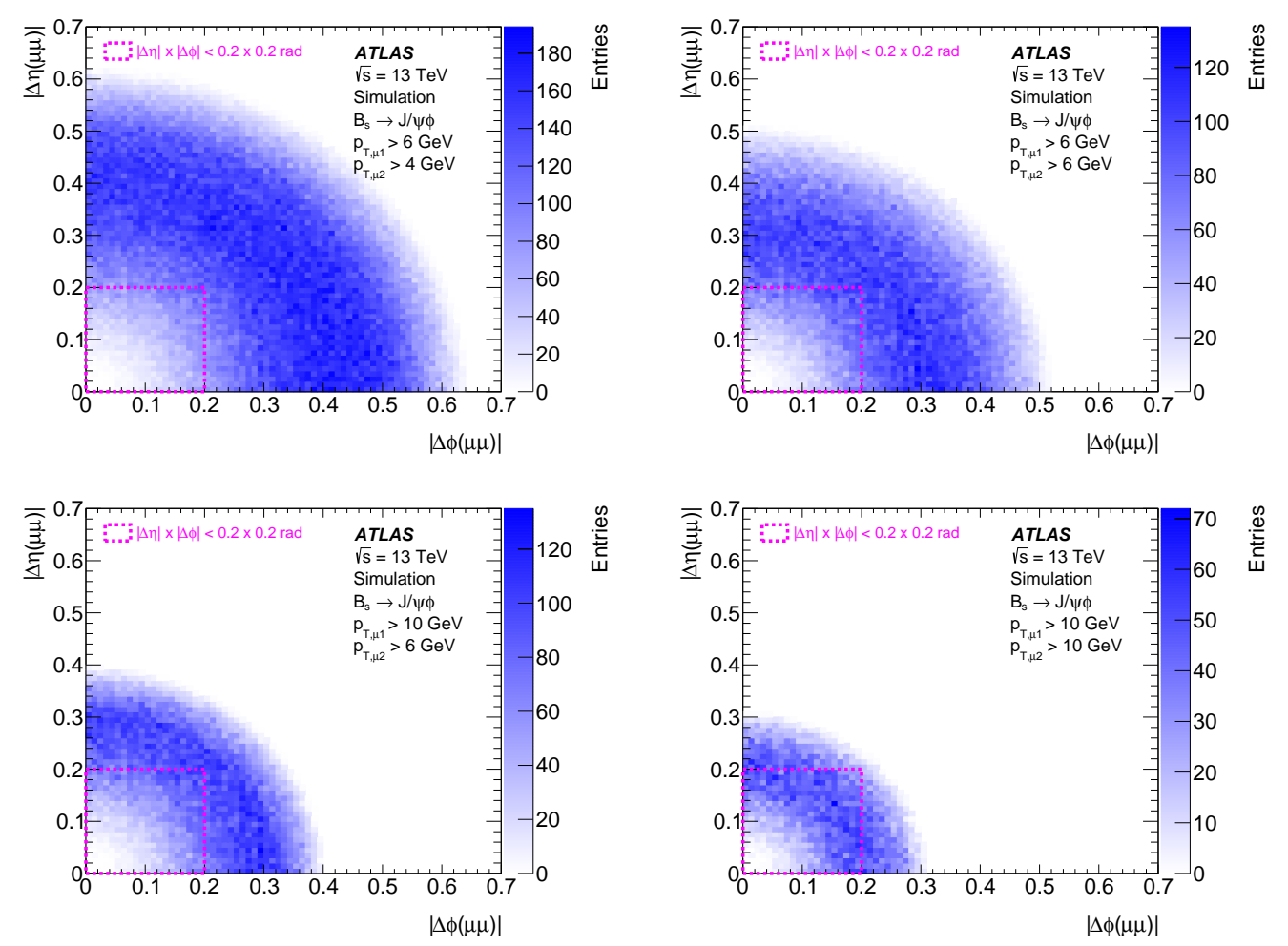

Figure 2: Angular separation of muon candidates for $\mathrm{B}_{\mathrm{S}} \rightarrow \mathrm{J} / \Psi \phi$ decays as a function of dimuon trigger $p_{\mathrm{T}}$ thresholds [3].

A new trigger has been studied, in the context of the phase-II upgrade of the TDAQ system [3], to select multiple muon candidates in the same RoI. Adopting this new trigger, the sensitivity is increasing without increasing sensibly the overall trigger rate. The efficiency of this algorithm 
depends on the resolution parameter, the smaller the resolution the higher the efficiency. In the triggering algorithm the minimal angular di-muon resolution $\Delta \phi=0.01$ was used. Smaller resolutions were tested but could not be used as the fake rate was overwhelming.

As a benchmark the FRVZ vector portal model [2] was tested and an overall $10 \%$ gain in signal efficiency is predicted. During this test an efficiency gain dependency on the LLP lifetime is seen, this effect is currently under investigation.

The final step to this analysis will be the evaluation of the trigger rate and a more comprehensive study of the fake rate. Preliminary studies have estimated a rate of $17 \mathrm{kHz}$ for the new proposed trigger, compatible with the phase-II TDAQ system requirements.

\section{References}

[1] ATLAS Collaboration, 2008 JINST 3 S08003.

[2] A. Falkowski, J. T. Ruderman, T. Volansky and J. Zupan, Hidden higgs decaying to lepton jets, Journal of High Energy Physics 2010 (2010) 77.

[3] ATLAS Collaboration, ATLAS-TDR-029, https://cds.cern.ch/record/2285584. 